\title{
The Development of Foveolar-type Gastric Adenocarcinoma during Maintenance Therapy of Vonoprazan for Reflux Esophagitis
}

\author{
Masahiro Saito ${ }^{1,2}$, Tomoyuki Koike ${ }^{1}$, Yasuaki Abe ${ }^{1}$, Kenichiro Nakagawa ${ }^{1}$, Takeshi Kanno ${ }^{1}$, \\ Xiaoyi Jin ${ }^{1}$, Waku Hatta ${ }^{1}$, Kaname Uno ${ }^{1}$, Naoki Asano ${ }^{1}$, Akira Imatani ${ }^{1}$, \\ Fumiyoshi Fujishima $^{3}$ and Atsushi Masamune ${ }^{1}$
}

\begin{abstract}
:
We herein report the first case of foveolar-type gastric adenocarcinoma that developed after the initiation of vonoprazan (VPZ). A 51-year-old man had heartburn at the first visit and reflux esophagitis endoscopically, so he started taking VPZ. An approximately 5-mm-sized reddish polyp with a raspberry-like morphology was detected at the anterior wall of the upper body of the stomach 156 weeks after starting maintenance therapy with VPZ $10 \mathrm{mg} /$ day. It was diagnosed as foveolar-type gastric adenocarcinoma based on a biopsy. Another approximately 4-mm-sized foveolar-type gastric adenocarcinoma was also detected at the posterior wall of the middle body of the stomach.
\end{abstract}

Key words: potassium-competitive acid blocker (P-CAB), vonoprazan (VPZ), foveolar-type gastric adenocarcinoma, serum gastrin

(Intern Med 60: 391-396, 2021)

(DOI: 10.2169/internalmedicine.5431-20)

\section{Introduction}

Vonoprazan (VPZ) is a potassium-competitive acid blocker (P-CAB) that was developed and granted coverage by the public health insurance system in Japan in February 2015 (1). P-CABs are a class of drugs that reversibly inhibit gastric acid secretion by competing with $\mathrm{K}^{+}$in the gastric proton pump enzyme $\mathrm{H}^{+} / \mathrm{K}^{+}$-ATPase (2). VPZ is capable of stronger acid inhibition than conventional acid inhibitors [proton pump inhibitors (PPIs)] (3). It has been reported that VPZ is effective for gastric and duodenal ulcers (4), the eradication of Helicobacter pylori (Hp) (5), and the treatment of reflux esophagitis (6), but it induces higher levels of serum gastrin than conventional PPIs (7). However, the duration of these previous studies was too short to explore the long-term clinical implications of an elevated serum gastrin concentration (1). There are still many unclear points regard- ing the clinical effects of long-term administration of VPZ.

Thus far, no cases of gastric adenocarcinoma that developed during the maintenance administration of VPZ have been reported. We herein report a case of two lesions of foveolar-type gastric adenocarcinoma that newly appeared after the initiation of VPZ and during the maintenance therapy for reflux esophagitis with oral administration of VPZ $10 \mathrm{mg} /$ day.

\section{Case Report}

A 51-year-old man was referred to our hospital because of heartburn and abdominal discomfort. His medical history included hypertension and hyperlipidemia, and he took statin (rosuvastatin calcium $2.5 \mathrm{mg} /$ day) and calcium-channel blocker (azelnidipine $16 \mathrm{mg} /$ day). There was nothing noteworthy in the family history, and he had no history of drinking or smoking.

${ }^{1}$ Division of Gastroenterology, Tohoku University Graduate School of Medicine, Japan, ${ }^{2}$ Tohoku University Tohoku Medical Megabank Organization, Japan and ${ }^{3}$ Division of Pathology, Tohoku University Graduate School of Medicine, Japan 
Table. Changes in Gastrin and PG by VPZ Administration.

\begin{tabular}{lccccccc}
\hline & \multicolumn{2}{c}{$\begin{array}{c}\text { Initial treatment with } \\
\text { VPZ 20mg/day }\end{array}$} & \multicolumn{3}{c}{ Maintenance treatment with VPZ 10mg/day } \\
& At the first visit & 4 weeks & 24 weeks & 48 weeks & 108 weeks & 156 weeks & 169 weeks \\
\hline Gastrin & $\mathbf{1 5 1}$ & $\mathbf{6 6 2}$ & $\mathbf{7 1 8}$ & $\mathbf{7 2 9}$ & $\mathbf{9 6 6}$ & $\mathbf{8 0 4}$ & $\mathbf{6 8 3}$ \\
PG I & $\mathbf{5 7 . 0}$ & $\mathbf{1 , 2 1 3 . 0}$ & $\mathbf{5 3 3 . 4}$ & $\mathbf{2 1 8 . 3}$ & $\mathbf{3 0 0 . 5}$ & $\mathbf{1 8 1 . 8}$ & $\mathbf{2 4 1 . 3}$ \\
PG II & $\mathbf{1 0 . 5}$ & $\mathbf{2 0 3 . 0}$ & $\mathbf{8 5 . 7}$ & $\mathbf{3 0 . 7}$ & $\mathbf{5 1 . 0}$ & $\mathbf{2 6 . 0}$ & $\mathbf{3 0 . 1}$ \\
PGI/PGII ratio & $\mathbf{5 . 4}$ & $\mathbf{6 . 0}$ & $\mathbf{6 . 2}$ & $\mathbf{7 . 1}$ & $\mathbf{5 . 9}$ & $\mathbf{7 . 0}$ & $\mathbf{8 . 0}$ \\
\hline
\end{tabular}

VPZ: vonoprazan PG: pepsinogen

There were no remarkable physical findings and nothing especially noteworthy regarding the blood test findings at the first visit. Hp was negative according to serum anti-Hp IgG antibody (E Plate "Eiken" H. pylori antibody; Eiken Chemical, Tokyo, Japan), urea breath test (Ubit ${ }^{\circledR}$; Otsuka Pharmaceutical, Tokyo, Japan), and a histological examination of a biopsy specimen taken from the gastric body mucosa. Hp eradication therapy had not previously been provided. Serum gastrin was measured by Gastrin RIA Kit II (FUJIREBIO, Tokyo, Japan). The serum pepsinogen (PG) I and PG II level were measured by Auto pepsinogen I BML$2 \mathrm{G}$ and Auto pepsinogen II BML-2 G (BML, Tokyo, Japan), respectively. Before the start of oral treatment, the serum gastrin level was 151, the serum PG I level was 57.0, and the PG II level was 10.5 (Table). The frequency scale for the symptoms of gastroesophageal reflux disease (FSSG) score at the first visit was 12 . He had no other symptoms besides heartburn and abdominal discomfort.

Endoscopy revealed no atrophic changes in the gastric mucosa but did detect reflux esophagitis, classified as Los Angeles classification Grade B. A regular arrangement of collecting venules (RAC) on the lesser curvature of the gastric angle was observed. No atrophy was observed in the gastric mucosa. There were small fundic gland polyps (FGPs) in the gastric body and fornix.

The patient started to take VPZ $20 \mathrm{mg}$ /day orally. After continued oral administration for four weeks, the symptoms almost disappeared, and the FSSG score improved to 3 . Follow-up endoscopy was performed, and healing of the reflux esophagitis was observed. The FGPs showed no marked change from the first endoscopy procedure. Therefore, we shifted from the initial treatment period to maintenance therapy for reflux esophagitis, and the dose of VPZ was changed from $20 \mathrm{mg}$ to $10 \mathrm{mg} /$ day. Despite dose reduction, no residual symptoms were observed. Forty-eight weeks after the start of maintenance therapy with VPZ $10 \mathrm{mg} /$ day, the patient underwent endoscopy. The reflux esophagitis remained cured, FGPs were unchanged, and no other abnormal findings were found. Furthermore, follow-up endoscopy was performed at 108 and 130 weeks after the initiation of maintenance therapy. FGPs were also unchanged, and no other abnormal findings were found.

The patient was examined again 156 weeks after the initiation of maintenance therapy. Endoscopy revealed an approximately 5 -mm-sized reddish polyp with a raspberry-like morphology in the anterior wall of the upper body of the stomach (Fig. 1E, F). The lesion was recognized as a brownish polyp on narrow-band imaging (NBI), and the surface of the lesion had a papillary structure (Fig. 2). This lesion was different from the FGPs, and a biopsy was performed. The biopsy indicated that the dysplastic foveolar epithelium showed tubular and papillary growth with architectural atypia, and the nuclei were round to oval in shape with disturbed polarity (Fig. 3). Immunohistochemistry showed that the neoplastic cells were positive for MUC5AC expression and negative for MUC6, MUC2, and CD10 expression. Ki-67 was over-expressed (labeling index was about $70 \%$ ), and positive cells were irregularly distributed on the mucosal surface. p53 over-staining was not seen. (Fig. 4). The lesions were diagnosed as foveolar-type gastric adenocarcinoma. When the endoscopic images were retrospectively reviewed, this lesion was able to be detected as a small reddish polyp at 130 and 108 weeks from the initiation of the maintenance therapy (Fig. 1C, D). However, at 48 weeks from the start of maintenance therapy and the first endoscopy, no mucosal changes were observed (Fig. 1A, B). On endoscopy performed 8 weeks later (164 weeks after the initiation of the maintenance therapy), the lesion had disappeared due to the biopsy.

An additional biopsy was performed at the scar of the previous biopsy, but no residual cancer was observed. At the same time, another reddish polyp was noted at the posterior wall of the middle body of the stomach. The size of this lesion was about $4 \mathrm{~mm}$, and the endoscopic findings were almost the same as those of the first detected lesion. The surface structure of the polyp was reddish with a papillary structure, showing raspberry-like appearance (Fig. 5C, D). Magnified observation with blue light imaging (BLI) revealed a papillary structure with a circular surface microstructure. The surface micro-vessels exhibited a dilated open loop with differences in caliber (Fig. 6). The pathological findings of the lesion also indicated dysplastic foveolar epithelium showing tubular and papillary growth with architectural atypia, and the nuclei were round to oval in shape with disturbed polarity (Fig. 7). Immunohistochemistry showed that the neoplastic cells were positive for MUC5AC expression and negative for MUC6, MUC2, and CD10 expression. This lesion was also diagnosed as foveolar-type gastric adenocarcinoma by a biopsy. When the endoscopic image was retrospectively reviewed for the second lesion, an extremely 


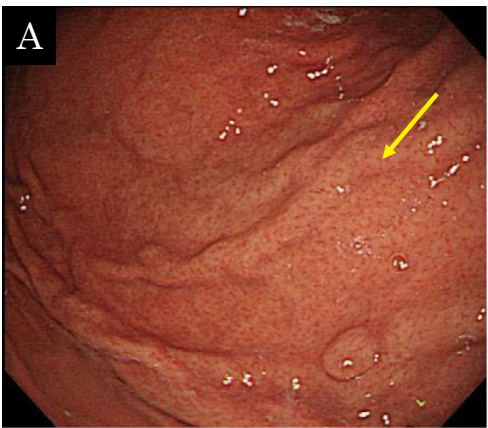

First endoscopy

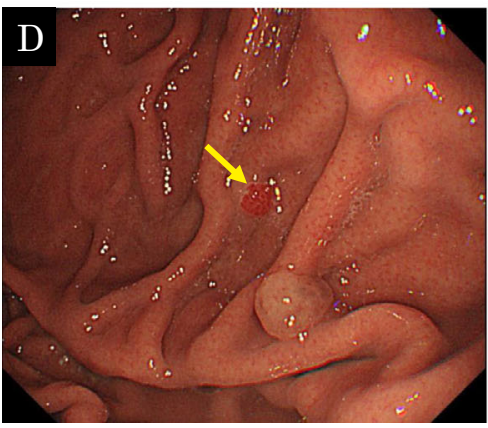

130W

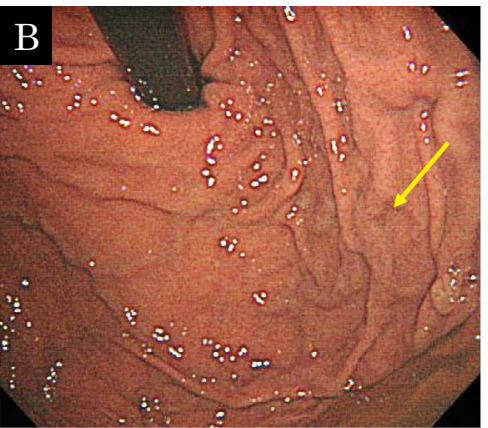

48W

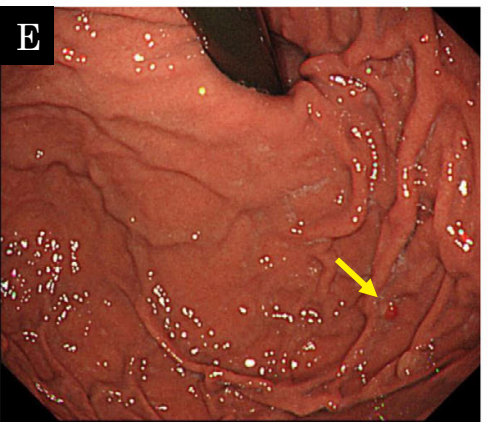

156W

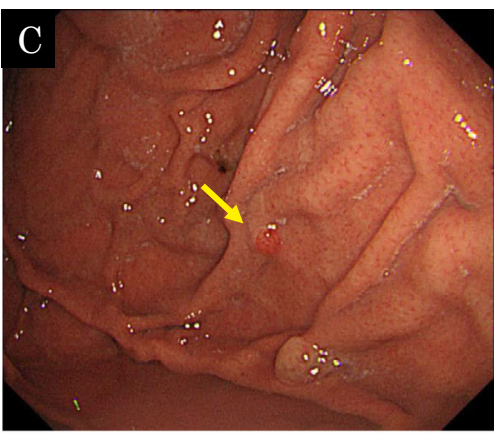

108W

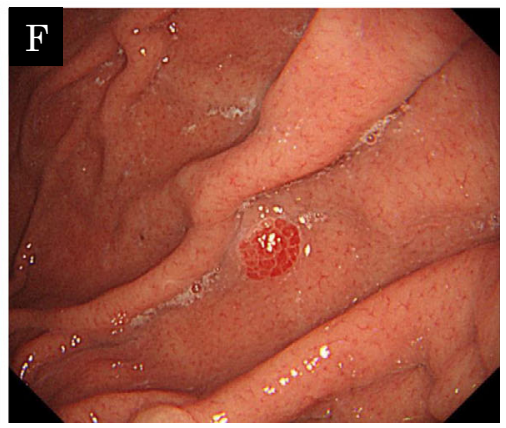

Figure 1. Time series of endoscopic images of the first lesion. 'Weeks' in the figure refer to the time after the initiation of maintenance therapy. At the first endoscopy procedure, no mucosal changes were observed (A). At 48 weeks from the start of the maintenance therapy, no mucosal changes were observed (B). When the endoscopic image was retrospectively reviewed, this lesion was recognized as a small reddish polyp at 108 weeks after the initiation of the maintenance therapy (C). When the endoscopic image was retrospectively reviewed, this lesion was recognized as a small reddish polyp at 130 weeks after the initiation of the maintenance therapy (D). An approximately 5-mm-sized reddish polyp was detected at the anterior wall of the upper gastric body on the endoscopy findings examined 156 weeks after the initiation of the maintenance therapy. The lesion showed a raspberry-like morphology (E, F).

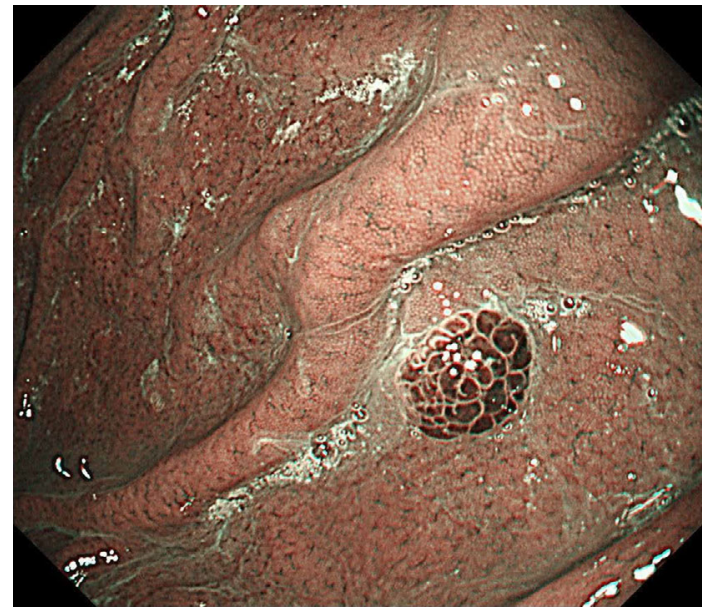

Figure 2. Narrow-band imaging (NBI) with near-focus magnification showed a brownish polyp, and the surface of the lesion had a papillary structure.

small red polyp was found at 130 weeks after the initiation of the maintenance therapy (Fig. 5B). However, no lesion was observed at 108 weeks after the start of maintenance therapy (Fig. 5A). Endoscopy was performed again 5 weeks later (168 weeks after the initiation of the maintenance therapy), and the lesion had also already disappeared following the biopsy. A biopsy was performed at the scar of the biopsy, and no residual cancer was observed.

Serum gastrin levels were followed up during maintenance therapy. Before the administration of VPZ, the levels were in the normal range at $151 \mathrm{pg} / \mathrm{mL}$, but they increased to $662 \mathrm{pg} / \mathrm{mL}$ at 4 weeks after the administration of VPZ 20 $\mathrm{mg} /$ day and remained high at about $700 \mathrm{pg} / \mathrm{mL}$. Both PG I and PG II values also increased after the oral administration of VPZ (Table).

There were no endoscopic findings suggestive of remnant or residual cancer. We are therefore continuing to follow the patient with endoscopy.

\section{Discussion}

This is the first case report in which the development of foveolar-type gastric adenocarcinomas was detected during 

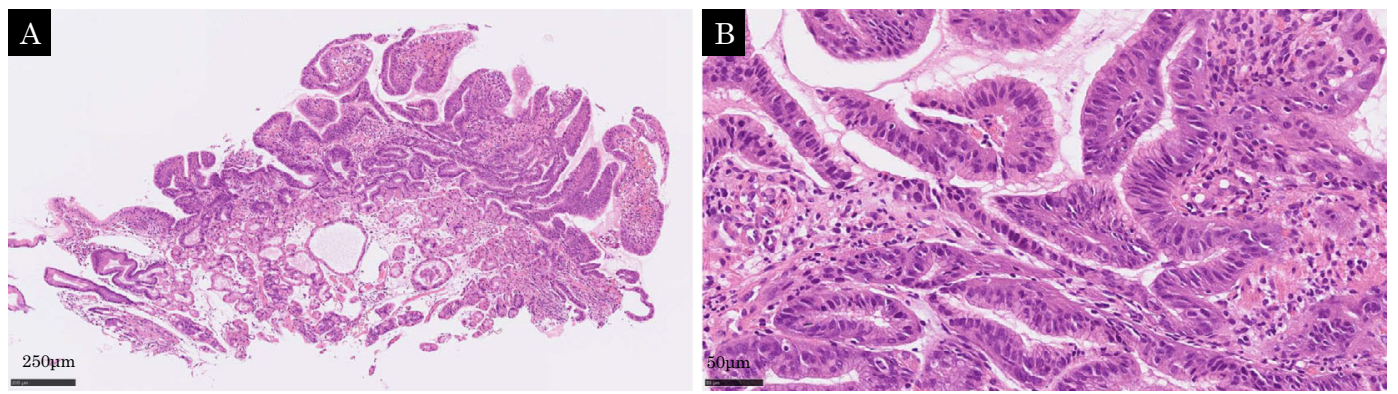

Figure 3. The lesion was diagnosed as foveolar-type gastric adenocarcinoma by a biopsy at 156 weeks after the initiation of the maintenance therapy. Dysplastic foveolar epithelium showed tubular and papillary growth with architectural atypia, and the nuclei were round to oval in shape with disturbed polarity (A, B).
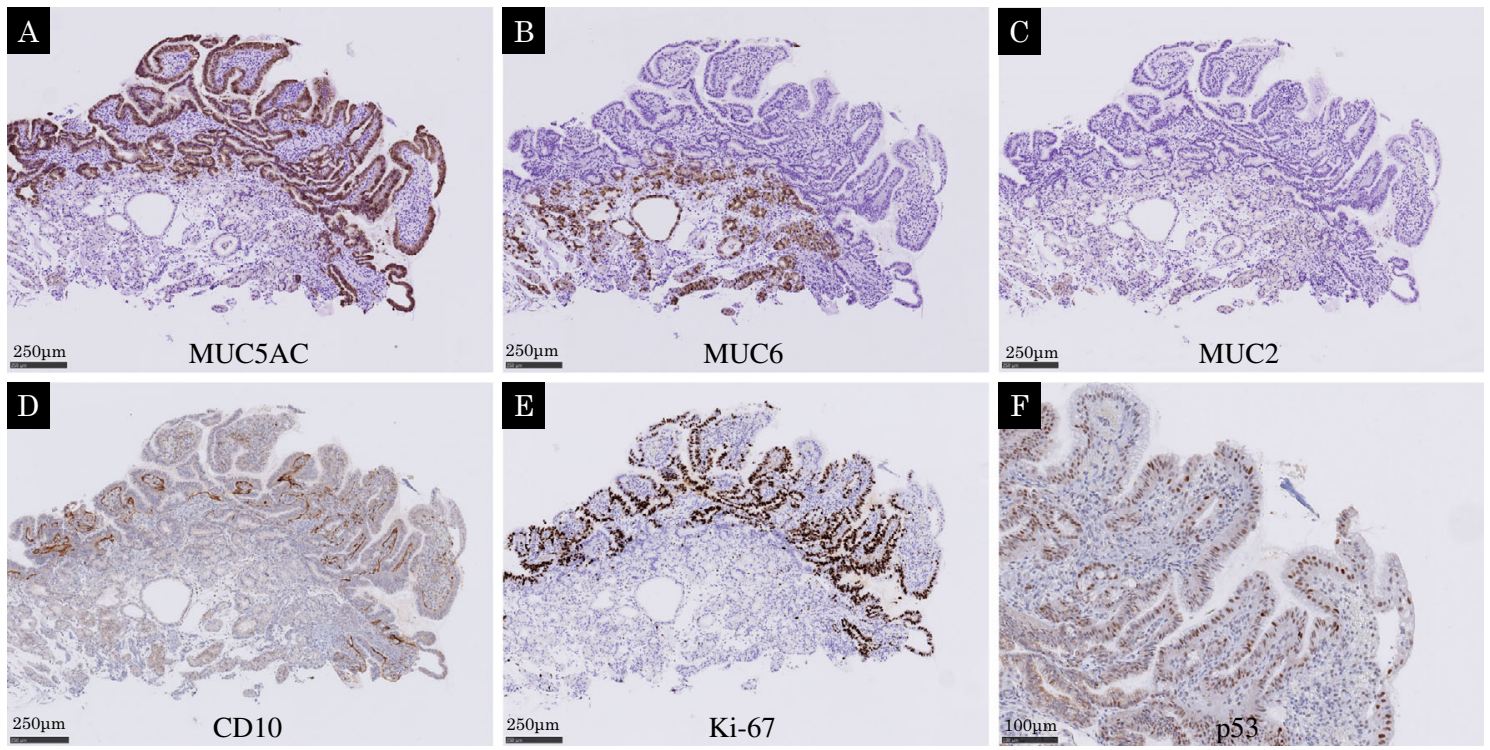

Figure 4. Immunohistochemistry showed that neoplastic cells were positive for MUC5AC (A) and negative for MUC6 (B), MUC2 (C), and CD10 (D). Ki-67 was over-expressed (labeling index was about $70 \%$, and positive cells were irregularly distributed on the mucosal surface $(\mathrm{E})$. p53 overstaining was not seen $(F)$.

VPZ administration. In this case, the development of two lesions of gastric cancer was confirmed during maintenance therapy of VPZ for reflux esophagitis. Neither lesion existed at the start of VPZ administration but newly developed and tended to grow over the course of treatment. Hp negativity was confirmed by the urea breath test and serum antibody before the start of VPZ, although the patient had no history of eradication. The surrounding gastric mucosa was RACpositive mucosa that was not infected with $\mathrm{Hp}$.

VPZ is effective for treating reflux esophagitis due to its strong inhibitory effect on acid secretion (6) and was approved for use in the public health insurance system in Japan from February 2015 (1). It was reported that the gastrin level increases during conventional PPI therapy (8), and there are concerns about a greater increase in gastrin levels and an increased risk of gastric mucosal hyperplasia with VPZ than with conventional PPIs (9). Previous studies in mice have suggested that hypergastrinemia associated with
PPIs may promote gastric cancer through the gastrin receptor $(10,11)$. However, we do not have enough data about long-term adverse events, such as the risk of neuroendocrine tumors (NET) or gastric cancer due to elevated gastrin levels, during VPZ treatment. There have thus far been no reports of gastric cancer developing during P-CAB administration. While the presence of a relationship between the oral administration of P-CABs and the development of cancer is unclear, this was the first case in which foveolar-type gastric adenocarcinoma was found to develop during the administration of a P-CAB as maintenance therapy for reflux esophagitis.

Foveolar-type adenocarcinoma is a very rare tumor showing a raspberry-like appearance that develops in Hpuninfected stomach. Its diagnosis is based on the Japanese Classification of Gastric Carcinoma (JCGC), although Western criteria have described histologic characteristics similar to those of foveolar-type adenoma $(12,13)$. 


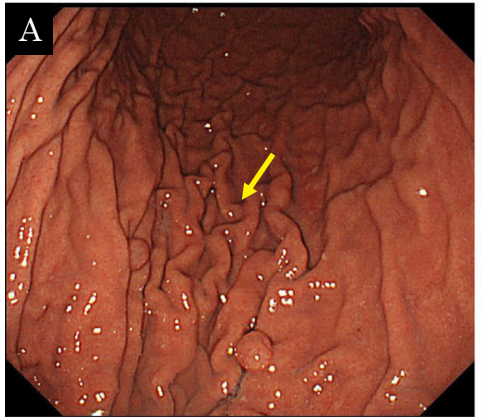

$108 \mathrm{~W}$

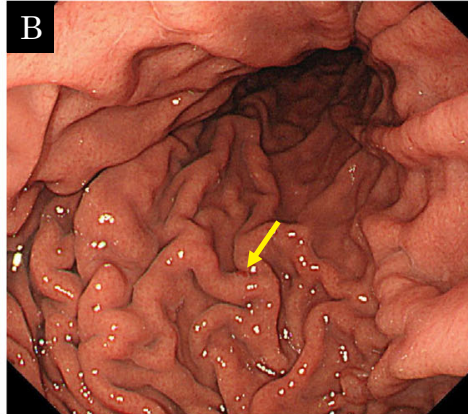

130W

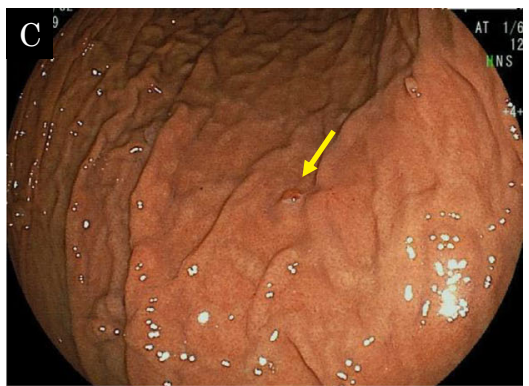

$\mathrm{D}$

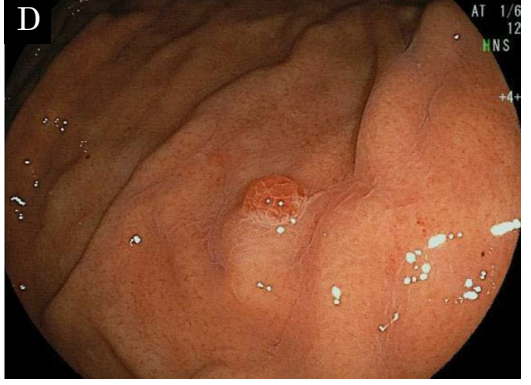

$164 \mathrm{~W}$

Figure 5. Time series of endoscopic images of the second lesion. Weeks in the figure are the weeks after the initiation of maintenance therapy. When the endoscopic image was retrospectively reviewed for the second lesion, no lesion was observed at 108 weeks after the initiation of the maintenance therapy (A). When the endoscopic image was retrospectively reviewed for the second lesion, an extremely small red polyp was found at 130 weeks after the initiation of the maintenance therapy (B). Another reddish polyp was noted at the posterior wall of the middle body of the stomach 164 weeks after the initiation of the maintenance therapy (C, D). The size of this lesion was about $4 \mathrm{~mm}$, and the endoscopic findings were almost the same as those of the first detected lesion. The surface structure of the polyp was reddish with a papillary structure, showing a raspberry-like appearance.

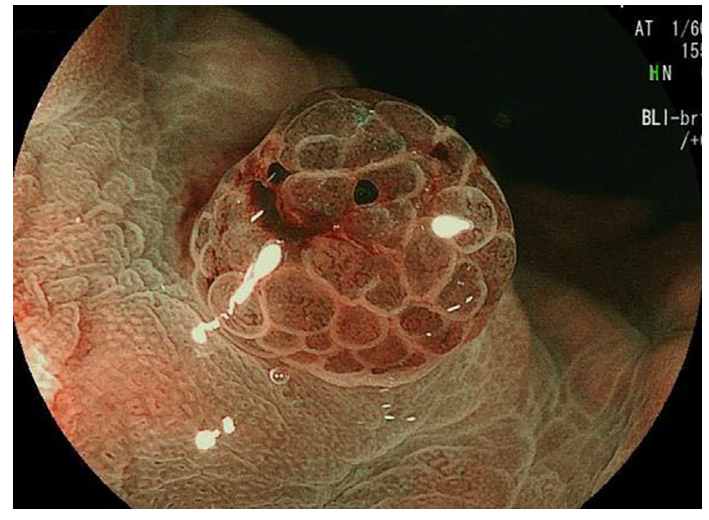

Figure 6. The magnified observation with blue light imaging (BLI) revealed a papillary structure with a circular surface microstructure. The surface micro-vessels exhibited a dilated open loop with differences in the caliber.

Shibagaki et al. found that, among 14 patients with raspberry-like foveolar-type adenocarcinoma according to the JCGC, 13 had not taken any PPIs, and the gastrin levels were within the normal range, except for in 1 patient who had been administered a PPI (13). Consequently, higher gastrin levels were considered unlikely to be involved as the carcinogenic factor of raspberry-like foveolar-type gastric adenocarcinoma. In the present case, the serum gastrin level

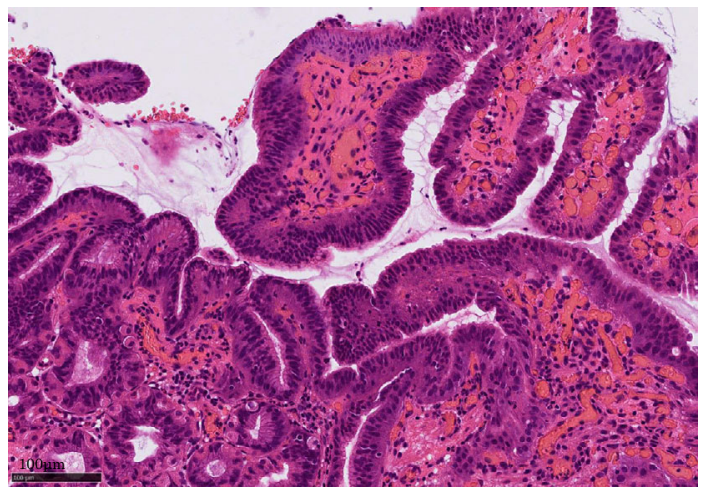

Figure 7. The pathology of the second lesion according to the biopsy is shown. Dysplastic foveolar epithelium showed tubular and papillary growth with architectural atypia, and the nuclei were round to oval in shape with disturbed polarity. This lesion was also diagnosed as foveolar-type gastric adenocarcinoma.

remained consistently high after starting the oral administration of VPZ. Although no relationship between an increased level of gastrin and the development of tumors has been established, it is understandable that a continuously high gastrin level might affect the development of foveolar-type gastric adenocarcinoma.

The tumorigenic mechanism underlying the development 
of gastric cancer in an Hp-uninfected population is unclear at present, aside from cases of hereditary diffuse gastric cancer, which occurs regardless of HP infection based on germline mutations in the epithelial cadherin gene CDH1 $(13,14)$. The present patient had no family history of gastric cancer.

P-CABs have good therapeutic effects for reflux esophagitis, but it may be necessary to regularly check for changes in the gastric mucosa during long-term administration. Further clinical trials concerning the long-term safety and tolerability of VPZ are necessary. At present, a randomized controlled trial is underway to investigate the safety of the long-term use of VPZ for reflux esophagitis. We will have to wait for the results of this study in order to evaluate the long-term efficacy and adverse events of VPZ (9).

\section{Author's disclosure of potential Conflicts of Interest (COI).}

Tomoyuki Koike: Honoraria, Takeda Pharmaceutical. Atsushi Masamune: Honoraria, Otsuka Pharmaceutical.

\section{References}

1. Yang X, Li Y, Sun Y, et al. Vonoprazan: a novel and potent alternative in the treatment of acid-related diseases. Dig Dis Sci 63: 302-311, 2018.

2. Garnock-Jones KP. Vonoprazan: first global approval. Drugs $\mathbf{7 5}$ 439-443, 2015.

3. Sakurai Y, Mori Y, Okamoto H, et al. Acid-inhibitory effects of vonoprazan $20 \mathrm{mg}$ compared with esomeprazole $20 \mathrm{mg}$ or rabeprazole $10 \mathrm{mg}$ in healthy adult male subjects - a randomised open-label cross-over study. Aliment Pharmacol Ther 42: 719-730, 2015.

4. Miwa H, Uedo N, Watari J, et al. Randomised clinical trial: efficacy and safety of vonoprazan vs. lansoprazole in patients with gastric or duodenal ulcers -results from two phase 3, noninferiority randomised controlled trials. Aliment Pharmacol Ther 45: 240-
252, 2017.

5. Jung Y, Kim E, Park C. Systematic review with metaanalysis: the efficacy of vonoprazan-based trial therapy on Helicobacter pylori eradication. Aliment Pharmacol Ther 46: 106-114, 2017.

6. Ashida K, Sakurai Y, Nishimura A, et al. Randomised clinical trial: a dose-ranging study of vonoprazan, a novel potassiumcompetitive acid blocker, vs. lansoprazole for the healing of erosive oesophagitis. Aliment Pharmacol Ther 43: 240-251, 2016.

7. Kagami T, Sahara S, Ichikawa H, et al. Potent acid inhibition by vonoprazan in comparison with esomeprazole, with reference to CYP2C19 genotype. Aliment Pharmacol Ther 43: 1048-1059, 2016.

8. Lundell L, Vieth M, Gibson F, et al. Systematic review: the effects of long-term proton pump inhibitor use on serum gastrin levels and gastric histology. Aliment Pharmacol Ther 42: 649-663, 2015.

9. Uemura N, Kinoshita Y, Haruma K, et al. Rationale and design of the VISION study: a randomized, open-label study to evaluate the long-term safety of vonoprazan as maintenance treatment in patients with erosive esophagitis. Clin Exp Gastroenterol 11: 51-56, 2018.

10. Hayakawa Y, Chang W, Jin G, et al. Gastrin and upper GI cancers. Curr Opin Pharmacol 31: 31-37, 2016.

11. Schmitz F, Goke MN, Otte JM, et al. Cellular expression of CCK$\mathrm{A}$ and CCK-B/gastrin receptors in human gastric mucosa. Regul Pept 102: 101-110, 2001.

12. Japanese Gastric Cancer Association. Japanese gastric cancer treatment guideline 2010: 3rd English edition. Gastric Cancer 14: 113123,2011

13. Shibagaki K, Fukuyama C, Mikami H, et al. Gastric foveolar-type adenomas endoscopically showing a raspberry-like appearance in the Helicobacter pylori -uninfected stomach. Endosc Int Open 7: E784-E791, 2019.

14. Hansford S, Kaurah P, Li-Chang H, et al. Hereditary diffuse gastric cancer syndrome: $\mathrm{CDH} 1$ mutations and beyond. JAMA Oncol 1: 23-32, 2015.

The Internal Medicine is an Open Access journal distributed under the Creative Commons Attribution-NonCommercial-NoDerivatives 4.0 International License. To view the details of this license, please visit (https://creativecommons.org/licenses/ by-nc-nd/4.0/).

(C) 2021 The Japanese Society of Internal Medicine Intern Med 60: 391-396, 2021 